\title{
Design and Implementation of Library Management System
}

\author{
Acheampong Samuel \\ Nanjing University of Post and \\ Telecommunication \\ China
}

\author{
Ampofo Godfred \\ Nanjing University of Post and \\ Telecommunication \\ China
}

\author{
$\mathrm{Xu} \mathrm{He}$ \\ Professor \\ Nanjing University of Post and \\ Telecommunication \\ China
}

\begin{abstract}
The Library Management System is an application for assisting

a librarian in managing a book library in a university. The system would provide basic set of features to add/update members, add/update books, and manage check in specifications for the systems based on the client's statement of need. Library management system is a typical management Information system (MIS), its Development include the establishment and maintenance of back-end database and front-end application development aspects. For the former require the establishment of data consistency and integrity of the strong data security and good libraries. As for the latter requires the application fully functional, easy to use and so on.
\end{abstract}

\section{Keywords}

Management System, Data Security, management information System

\section{INTRODUCTION}

The project titled Library Management System is Library

Management software for monitoring and controlling the transactions in a library. The project "Library Management System" is developed in php, which mainly focuses on basic operations in a library like adding new books, and updating new information, searching books and members and return books.

This project of "LIBRARY MANAGEMENT" of gives us the complete information about the library. We can enter the record of new books and retrieve the details of books available in the library. We can issue the books to the students and maintain their records and can also check how many books are issued and stock available in the library. In this project we can maintain the late fine of students who returns the issued books after the due date.

Throughout the project the focus has been on presenting information and comments in an easy and intelligible manner.
The project is very useful for those who want to know about Library Management System.

Library is regarded as the brain of any institute; many institutes understand the importance of the library to the growth of the institute and their esteem users (students). LMS support the general requirement of the library like acquisition, cataloging, circulation.

Library project system that offers many flexible and convenient features, allowing librarians and library users to maximize time and efficiency. Library System gives the all detailed information about students, staff and books. It will track on the how many books available in library and books issued to the students. It shows popular book among the students. It will provide book lost in library. It keeps the record of the suppliers and book binders. It generates MIS reports for management. Our software is customizable for any library requirement.

\section{METHOD AND MATERIALS}

\subsection{Development Methodology}

In software engineering, a system development methodology refers to the framework that is used to structure, plan, and control the process of developing an information system. Software development methodologies define the processes we use to build software. These methodologies are also referred to as Software Development Process Models. Each methodology follows a series of steps unique to its type, to ensure success in the process of software development.

A software process is a set of related activities that leads to the production of a software product.

There are a lot of software processes but they all include four activities:

$>$ Specification

$>$ Development 
$>$ Validation

$>$ Evolution

These are fundamental to software engineering. A wide variety of Software Development methodology has evolved over the years. Each of these methodologies has its own recognized strengths and weaknesses. The following are the most widely used methodologies for software development.

\subsubsection{Waterfall Method}

The waterfall model is a sequential approach, where each fundamental activity of a process represented as a separate phase, arranged in linear order. This model requires planning and scheduling activities before starting working on them, it is plan-driven.

Plan-driven process is one in which all the activities are first planned, and then each progress is measured against the plan.

The Agile process on the other hand involves planning incrementally and it is much easier to change the processes to reflect a change in requirement.

\subsubsection{Incremental Method}

The Incremental method of development is based on the idea of developing an initial implementation, exposing this model to user to receive feedback. This model evolves in the form of versions as the requirements change until an acceptable system has been developed.

\subsubsection{Spiral Method}

The spiral model is a risk-driven method where the process is represented as spiral rather than a sequence of activities and it includes best features from the waterfall and prototyping models. In addition, it introduces a new component called: risk-assessment. Each loop in the spiral represents a phase, thus the first loop might be concerned with system feasibility. The next loop might be concerned with the requirements definition and the next with system design, and the like.

\subsubsection{Prototyping Method}

A prototype is a version of a system or part of the system that's developed quickly to check the customer's requirements or the feasibility of some design decisions. Prototypes are useful when a customer or the developer is not sure of the requirements, the algorithms efficiency, some business rules or even response time.

The four basic process activities of specification, development, validation, and evolution are organized differently in the respective development processes. In the waterfall model, they are organized in sequence, but are interleaved in incremental development method. The type of software, the people, and organizational structures involved determines how these activities are carried out. For example, specifications for extreme programming are written on cards. Tests are executable and developed before the program itself. Evolution may involve substantial system restructuring or re-factoring.

This project uses the Incremental method in developing the application because the preferences of the users keep changing.

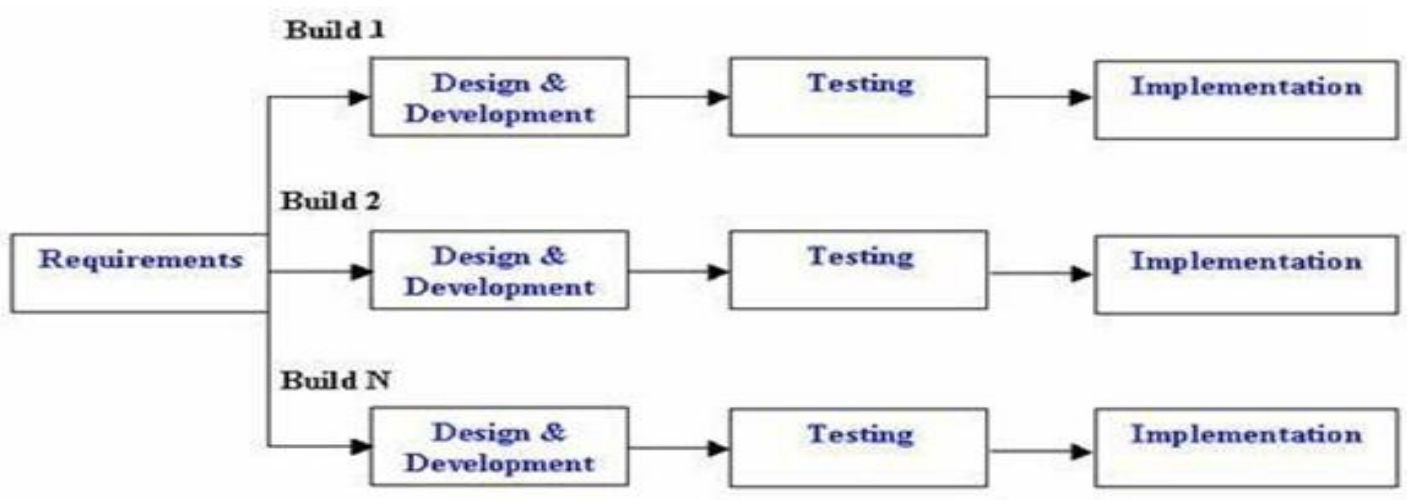

Figure 2.0 Incremental Development Methodology

\subsection{Database Design}

Database is any collection of data, or information, that is specially organized for rapid search and retrieval by a computer. Databases are structured to facilitate the storage, retrieval, modification, and deletion of data in conjunction with various data-processing operations. A database is stored 
as a file or a set of files on magnetic disk or tape, optical disk, or some other secondary storage device. The information in these files may be broken down into records, each of which consists of one or more fields. Fields are the basic units of data storage, and each field typically contains information pertaining to one aspect or attribute of the entity described by the database. Records are also organized into tables that include information about relationships between its various fields.

Database Design is a collection of processes that facilitate the designing, development, implementation and maintenance of enterprise data management systems. The main objectives of database designing are to produce logical and physical designs models of the proposed database system.

\subsection{Materials}

\section{Development Tools}

In this project, a number of development tools would be used to complete this project. They are listed as follow.

\section{Php}

PHP (recursive acronym for PHP: Hypertext Preprocessor)

is a widely-used open source general-purpose scripting language that is especially suited for web development and can be embedded into HTML. The best things in using PHP are that it is extremely simple for a newcomer, but offers many advanced features for a professional programmer. Don't be afraid reading the long list of PHP's features. You can jump in, in a short time, and start writing simple scripts in a few hours. What distinguishes PHP from something like client-side JavaScript is that the code is executed on the server, generating HTML which is then sent to the client. The client would receive the results of running that script, but would not know what the underlying code was. You can even configure your web server to process all your HTML files with PHP, and then there's really no way that users can tell what you have up your sleeve.

\section{HTML}

HTML (Hypertext Markup Language) is the code that is used to structure a web page and its content. HTML is used to specify whether a web content should be recognized as a paragraph, list, heading, link, image, multimedia player, form, or one of many other available elements or even a new element that you define. It is the globally accepted programming language for formatting web pages. It is mostly used by small and medium scale businesses that do not really need advanced functionality on their websites. HTML is free, supports all browsers on the client's machine, easy to use and understand hence, the choice in building the structure of my web pages.

\section{CSS}

Cascading Style Sheets (CSS) is a stylesheet language used to describe the presentation of a document written in HTML or XML (including XML dialects such as SVG or XHTML). CSS describes how elements should be rendered on screen, on paper, in speech, or on other media. CSS is one of the core languages of the open web and is standardized across browsers according to the W3C (World Wide Web Consortium) specification.

\section{JavaScript}

JavaScript is a high-level, dynamic, weakly typed, prototype-based, multi-paradigm, and interpreted programming language. JavaScript is a full-fledged dynamic programming language that, when applied to an HTML document, can provide dynamic interactivity on websites. It would be used in conjunction with Django to ensure validation rules on the front-end of the websites.

\section{Bootstrap}

Bootstrap is a free and open-source front-end web framework for designing websites and web applications. It contains HTML and CSS based design templates for typography, forms, buttons, navigation and other interface components, as well as optional JavaScript extensions. Unlike many web frameworks, it concerns itself with front-end development only. Bootstrap would be used to design the styling of the application alongside CSS. Bootstrap is important in the application for the following reasons:

$>$ Easy to use: Anybody with just basic knowledge of HTML and CSS can start using Bootstrap.

$>$ Responsive features: Bootstrap's responsive CSS adjusts to phones, tablets, and desktops.

> Mobile-first approach: Mobile-first styles are part of the core bootstrap framework.

D Browser compatibility: Bootstrap is compatible with all modern browsers (Chrome, Firefox, Internet Explorer, Safari, and Opera).

\section{Photoshop}

Adobe Photoshop is the predominant photo editing and 
manipulation software on the market. Its uses ranges from full featured editing of large batches of photos to creating intricate digital paintings and drawings that mimic those done by hand. It is a graphic designing tool that enables picture manipulation and editing. Photoshop would be used to design user interfaces and the various images that would be required in developing the system.

\subsection{Systems Analysis and Design}

System analysis and design deal with planning the development of information systems through understanding and specifying in detail what a system should do and how the components of the system should be implemented and work together. System analysis and design solve business problems through analyzing the requirements of information systems and designing such systems by applying analysis and design techniques.

System analysis and design is the most essential phase in the development of a system since the logical system design arrived at as a result of systems analysis which is in turn converted into physical system design.

\subsubsection{General System Architecture}

The architecture of applications is usually broken into logical chunks called "tiers", where every tier is assigned a role. A "tier" can also be referred to as a "layer". There are three layers involved in the application namely Presentation Layer, Business Layer and Data Layer. Each layer is explained in detailed below:

\section{$>$ Presentation Layer:}

It is also known as Client layer. Top most layer of an application. This is the layer we see when we use a software. By using this layer, we can access the webpages. The main functionality of this layer is to communicate with Application layer. This layer passes the information which is given by the user in terms of keyboard actions, mouse clicks to the Application Layer. For example, login page of Gmail where an end user could see text boxes and buttons to enter user id, password and to click on sign-in. In simple words, it is to view the application.

\section{Application Layer:}

It is also known as Business Logic Layer which is also known as logical layer. As per the Gmail login page example, once user clicks on the login button, Application layer interacts with Database layer and sends required information to the Presentation layer. It controls an application's functionality by performing detailed processing. This layer acts as a mediator between the Presentation and the Database layer. Complete business logic will be written in this layer. In simple words, it is to perform operations on the application.

\section{Data Layer:}

The data is stored in this layer. Application layer communicates with Database layer to retrieve the data. It contains methods that connects the database and performs required action e.g.: insert, update, delete etc. In simple words, it is to share and retrieve the data.

There are four categories of application architectures which all have the four layers explained above. The categories of the application architecture are:

\subsubsection{N-Tier Architecture}

It is similar to three tier architectures but number of application servers are increased and represented in individual tiers in order to distributed the business logic so that the logic will be distributed. N-Tier application is also known as

\section{Distributed application.}




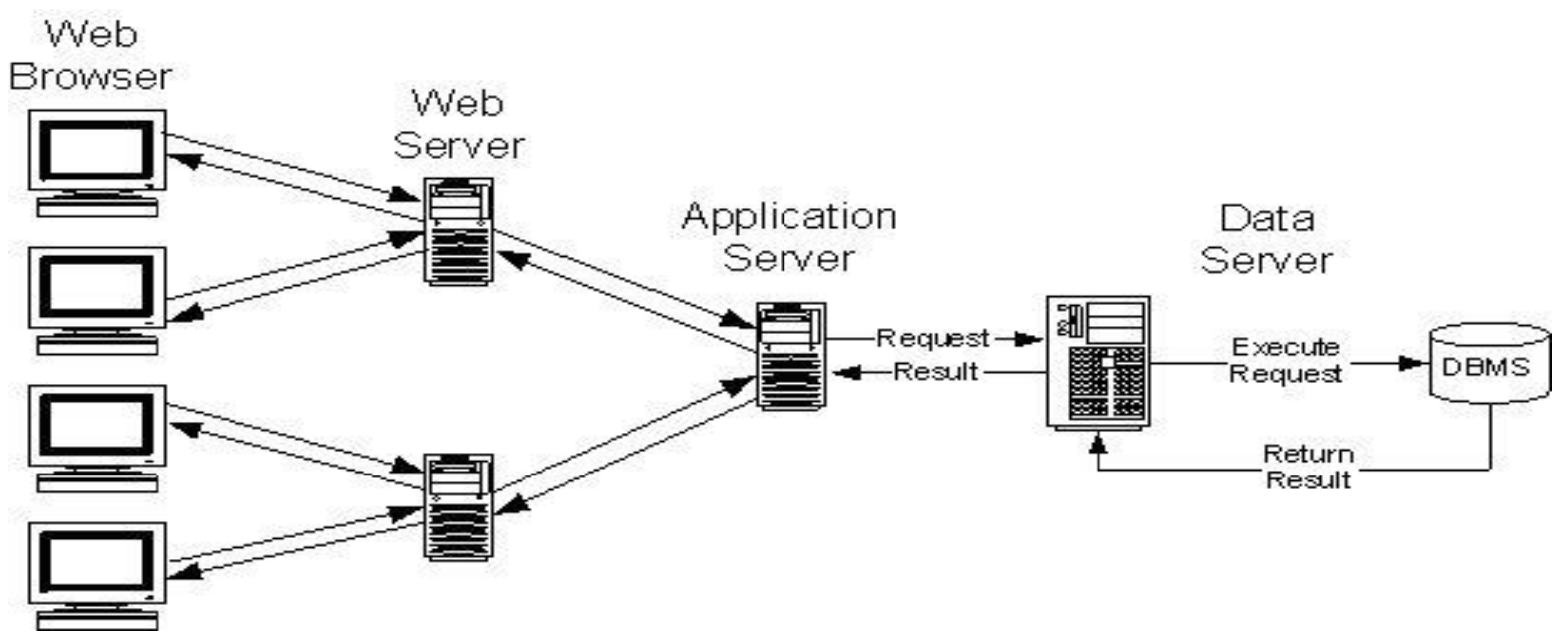

Figure 2.1 N-Tier Application (Distributed Application)

\section{ER Design}

Entity Relationship (ER) models have played a central role in systems specification, analysis and development. It is clear that the physical objects from the precious section: the borrower name, dye date, book name card, authors, etc., correspond to entities in the Entity Relationship model, and the operations to be done on those entities. however, a good design will minimize redundancy and attempt to store all the information in as small a space as possible. after some consideration, we have decided.

\section{E-R Diagram of Library Management System}

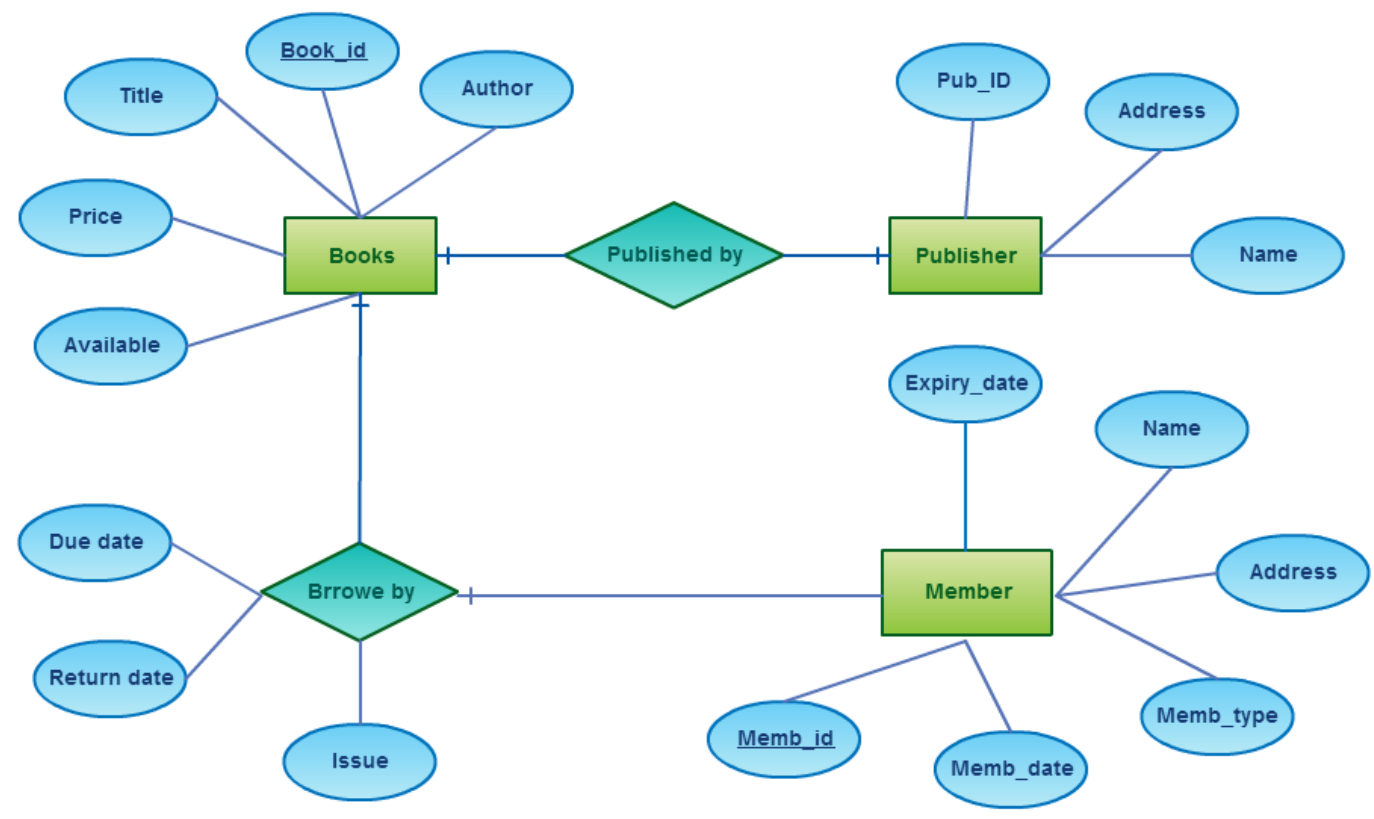

Figure 2.2 E-R Design

\section{RESULTS AND DISCUSSIONS}

After the system developed, process of system testing must be carry on in order to test if the system is free of bugs. If during the system testing, there are bugs or errors detected, the developer may need to correct and fix the bugs immediately.
Testing is the process of evaluating a software or system to detect differences between given input and expected output. Testing is conducted to assess the quality of a system hence can be said to be a validation and verification process. This ensures that system meets the technical requirements that guided its design and development, works as expected and can be 
implemented with the same characteristics.

There are few types of system testing that must be performed which include the unit testing, integration testing, system testing, and acceptance testing. System testing is not a testing that is limited only to the development team but it also requires the help from specific outsider (beta-tester) to test on the system acceptance.

\section{Some of the screen-shots after testing}

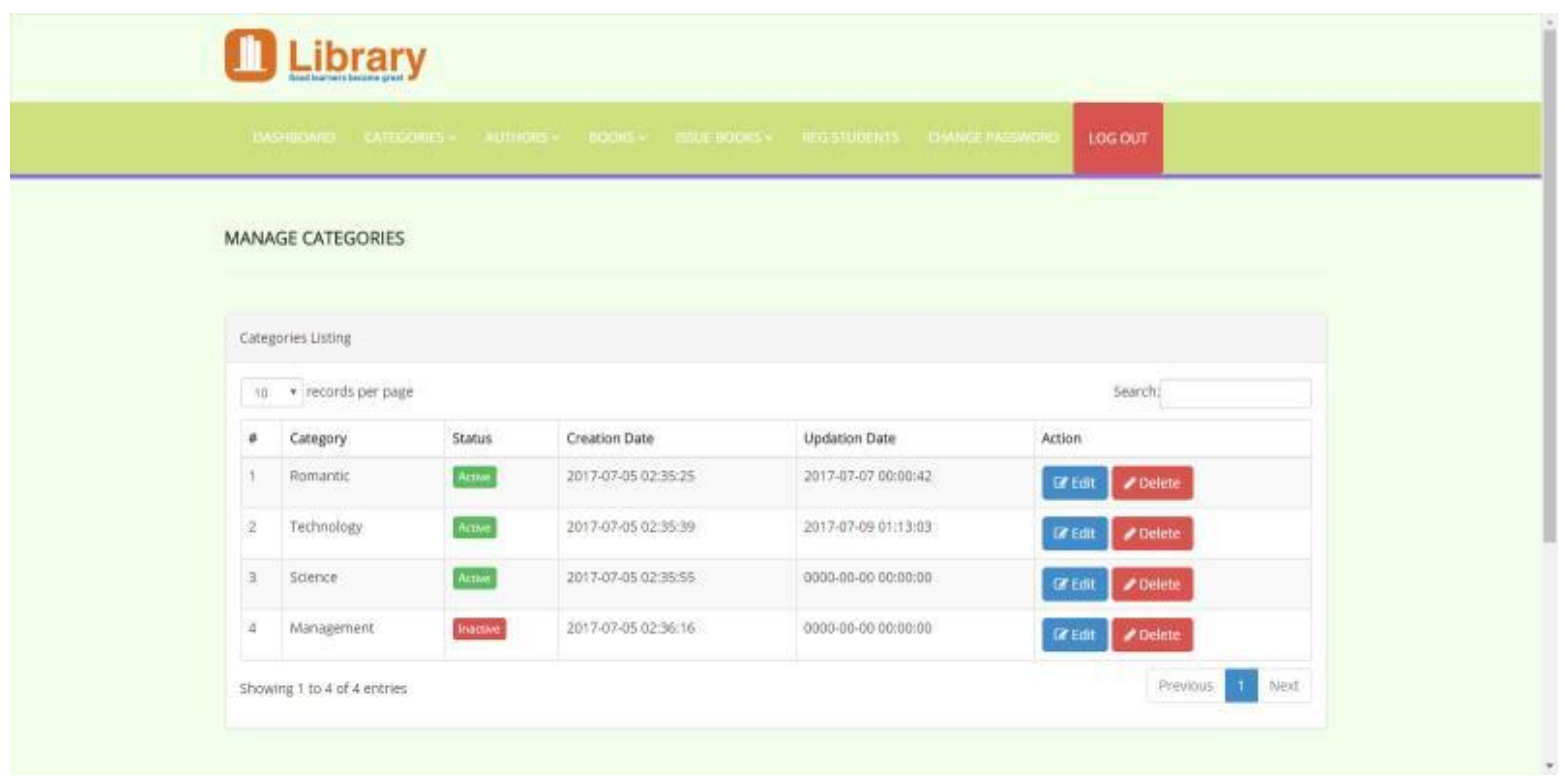

Figure 3.0 Manage Categories of Library Management System

\section{Q Library}

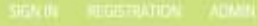

ADMIN 


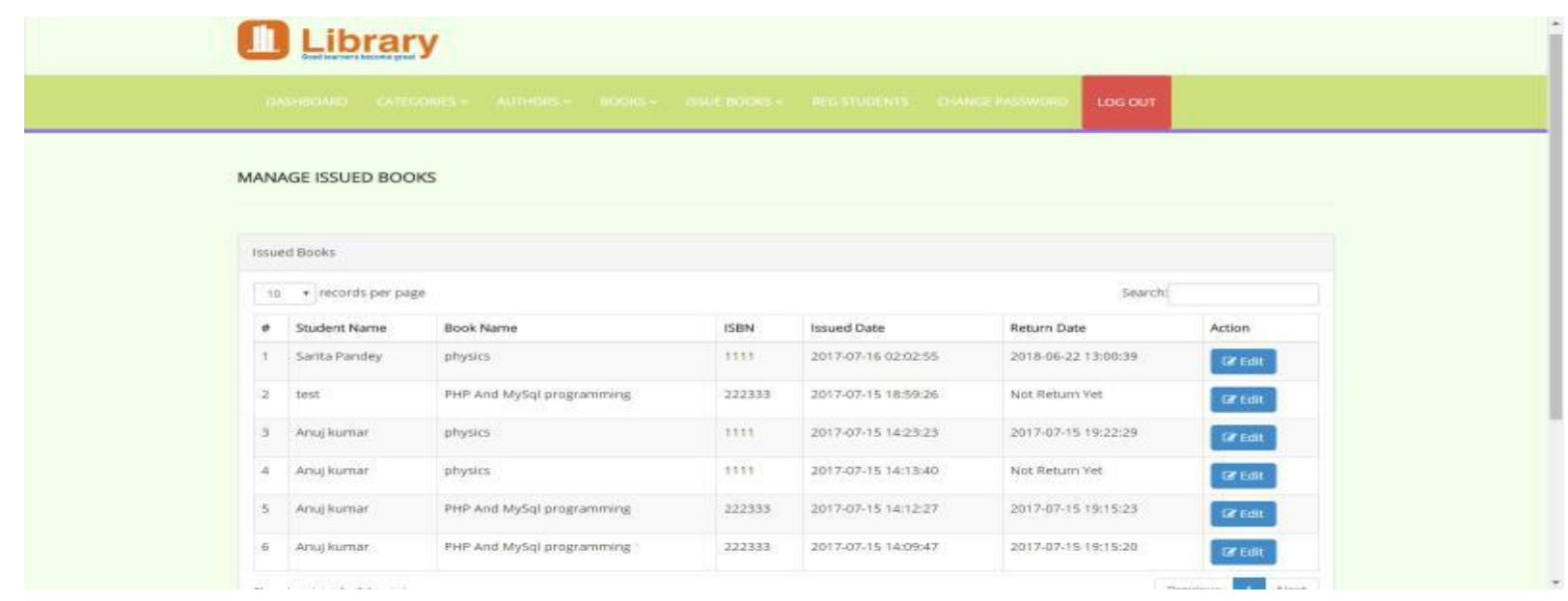

Figure 3.2 Manage Issued Books of the Library Management System

\section{CONCLUSION}

The Internet has become a major resource in modern life, thus library management system has gained significance not only from the management staff but also from the student's point of view. For the management, library system generates new books opportunities and for the student, it makes comparative selecting possible. As per a survey, most students of online library system are impulsive and usually make a decision to stay on a site within the first few seconds. Website design is like a management interior. If the management looks poor or like hundreds of other management the student is most likely to skip to the other site. Hence, the project has been designed to provide the user with easy navigation, retrieval of data and necessary feedback as much as possible

\section{FUTURE WORK}

In future, we hope to delve more into the integration of payments system in case one lost a book after borrowing. This is because the payment aspect of the system does not offer this to the user. The hope is to expand the choices available to the library management. Also, we hope to add multiple currency to make users choose the currency of their choice before they start any payment.

\section{ACKNOWLEDGMENTS}

We have learned a lot and really enjoyed working on this project. We thank God Almighty who by His Grace and Mercy we have been able to complete this project.

Our appreciation to our parents and guardians who have motivated us in diverse ways to come this far in our educational journey.

We are deeply indebted to our supervisor, Professor Xu He for his valuable guidance, stimulating suggestions, patience and for encouraging us to go ahead with our project. Thanks to them for his advice and useful feedback regarding the final project either in documentation parts or technical parts. In addition, their guideline and briefing on how to produce a good quality report for this project also provided to us. We are grateful.

We would like to sincerely thank all those who helped us with their valuable support during the entire process of this paper.

\section{REFERENCES}

[1] https://www.vendorview360.com/reference-library-mana gement/

[2] https://www.uef.fi/en/web/kirjasto/tietoa-kirjastosta/johta minen

[3] https://www2.illinois.gov/cdb/business/library/Document s/DCM2006.pdf

[4] http://thereferencelibrary.com/design_professionals/desig n_professionals.php

[5] http://thereferencelibrary.com/

[6] Nige, B. (2018, January 15). Why Django? Retrieved from Django Book: https://djangobook.com/tutorials/why-django/

[7] Rajkumar. (2018, April 05). Software Architecture: One-Tier, Two-Tier, Three Tier, N Tier. Retrieved from SoftwareTestingMaterial: https://www.softwaretestingmaterial.com/software-archit ecture/ 
[8] Sheppy. (2018, January 17). CSS: Cascading Style Sheets. Retrieved from MDN web docs: https://developer.mozilla.org/en-US/docs/Web/CSS

[9] SmartDraw. (2018, April 09). Entity Relationship Diagram. Retrieved from SmartDraw: https://www.smartdraw.com/entity-relationship-diagram/

[10] Smith, L. (2018, January 25). What PostgreSQL has over other open source SQL databases: Part I. Retrieved from Compose:

https://www.compose.com/articles/what-postgresql-has-o ver-other-open-source-sql-databases/

[11] studytonight. (2018, April 09). Normalization of Database. Retrieved from studytonight: https://www.studytonight.com/dbms/database-normalizat ion.php

[12] Team, P. C. (2017, November 23). 7 Key Features Online Shoppers Demand From an Online Store. Retrieved from PinnacleCart:

https://www.pinnaclecart.com/blog/7-key-features-online -shoppers-demand-from-an-online-store/

[13] The Editors of Encyclopaedia Britannica. (2018, April 06). Database. Retrieved from Britannica https://www.britannica.com/technology/database

[14] Wikipedia. (2018, April 09). User interface design. Retrieved from Wikipedia: https://en.wikipedia.org/wiki/User_interface_design 